\title{
THE 2-DIMENSIONAL CALABI FLOW
}

\author{
SHU-CHENG CHANG
}

\begin{abstract}
In this paper, based on a Harnack-type estimate and a local Sobolev constant bounded for the Calabi flow on closed surfaces, we extend author's previous results and show the long-time existence and convergence of solutions of 2-dimensional Calabi flow on closed surfaces. Then we establish the uniformization theorem for closed surfaces.
\end{abstract}

\section{$\S 1$. Introduction}

Let $\left(\Sigma, g_{0}\right)$ be a closed Riemann surface with a given conformal class $\left[g_{0}\right]$. In author's previous paper [Ch2], we consider the so-called Calabi flow on $\left(\Sigma,\left[g_{0}\right]\right)$ :

$$
\frac{\partial g_{i j}}{\partial t}=(\Delta R) g_{i j}, \quad g_{i j} \in\left[g_{0}\right] .
$$

If $g=e^{2 \lambda} g_{0}$, for a smooth function

$$
\lambda: \Sigma \times[0, \infty) \longrightarrow \mathbf{R},
$$

then the equations (1.1) reduce to the following initial value problem of fourth order parabolic equation on $\left(\Sigma,\left[g_{0}\right]\right)$

$$
\left\{\begin{array}{l}
\frac{\partial \lambda}{\partial t}=\frac{1}{2} \triangle R \\
\lambda(p, 0)=\lambda_{0}(p) \\
g=e^{2 \lambda} g_{0} \\
\int_{\Sigma} e^{2 \lambda_{0}} d \mu_{0}=\int_{\Sigma} d \mu_{0}
\end{array}\right.
$$

Received June 11, 2001.

Revised February 23, 2005, July 15, 2005.

2000 Mathematics Subject Classification: Primary 53C21; Secondary 58G03.

Research supported in part by NSC. 
where $\Delta=\Delta_{g}, \Delta_{0}=\Delta_{g_{0}}, R$ is the scalar curvature with respect to the metric $g, R_{0}$ is the scalar curvature with respect to the metric $g_{0}, d \mu_{0}$ is the volume element of $g_{0}$ and $d \mu$ is the volume element of $g$.

For the background metric $g_{0}$ with constant Gaussian curvature, P. T. Chruściel proved that the following result ([Chru]).

Proposition 1.1. Let $\left(\Sigma, g_{0}\right)$ be a Riemann surface with constant Gaussian curvature. For any given smooth initial value $\lambda_{0}$, there exists a smooth solution $\lambda(t)$ of $(1.2)$ on $\Sigma \times[0, \infty)$. Furthermore, the metric converges to a constant curvature metric.

Remark 1.1. Since there always exists a constant Gaussian curvature metric, due to the uniformization theorem in a Riemann surface, Chruściel's proof appears to be satisfactory for most purposes. However motivated by many reasons such as the study of higher-dimensional Calabi flow, it is desirable to remove this assumption. We refer to the author's review paper [Ch4] for more details. Moreover, X. X. Chen ([Chen]) has provided a new proof of Chruściel's result from such a motivation (from a viewpoint which is quite different from ours). But he still needed to assume the uniformization theorem.

Later, we proved the long-time existence and asymptotic convergence of solutions of $(1.2)$ on $\Sigma \times[0, \infty)$ for $\left(\Sigma, g_{0}\right)$ with $h=\operatorname{genus}(\Sigma) \geq 2$. Namely, we obtained the next result.

Proposition 1.2. ([Ch2]) Let $\left(\Sigma, g_{0}\right)$ be a closed surface of genus $h \geq$ 2 with any arbitrary background metric $g_{0}$. For any given smooth initial value $\lambda_{0}$, there exists a smooth solution $\lambda(t)$ of $(1.2)$ on $\Sigma \times[0, \infty)$. Furthermore, there exists a subsequence of solution, say $\lambda\left(t_{j}\right)$, such that $g=e^{2 \lambda\left(t_{j}\right)} g_{0}$ converges to the constant negative curvature metric $g_{\infty}$ as $t_{j} \rightarrow \infty$.

In this paper, we will extend Proposition 1.2 to more general cases. Our main results are the following two theorems.

THEOREM 1.3. Let $\left(\Sigma, g_{0}\right)$ be a closed Riemann surface of genus $h=$ 1. For any given smooth initial value $\lambda_{0}$, there exists a smooth solution $\lambda(t)$ of $(1.2)$ on $\Sigma \times[0, \infty)$. Furthermore, there exists a subsequence of solution $\lambda\left(t_{j}\right)$ such that $g=e^{2 \lambda\left(t_{j}\right)} g_{0}$ converges to a zero scalar curvature $g_{\infty}$ as $t_{j} \rightarrow \infty$. 
For a closed Riemann surface of genus $h=0$, there exists a metric $g_{1}$ with $R_{1}>0$. Moreover, from [KW, Lemma 6.1], it follows that there is a metric $g_{0}$ which is conformal equivalent to $g_{1}$ (i.e. $g_{0}$ is pointwise conformal to $\varphi^{*} g_{1}$ for some diffeomorphism $\varphi$ ) with $R_{0} \geq 0, R_{0} \neq 0$ and $R_{0}=0$ in a ball $B\left(p_{0}, \rho_{0}\right)$. Now we consider the Calabi flow on such a surface $\left(\Sigma,\left[g_{0}\right]\right)$.

THEOREM 1.4. Let $\left(\Sigma, g_{0}\right)$ be a closed Riemann surface of genus $h=0$ with $R_{0} \geq 0, R_{0} \neq 0$ and $R_{0}=0$ in a ball $B\left(p_{0}, \rho_{0}\right)$. For any given smooth initial value $\lambda_{0}$, there exists a smooth solution $\lambda(t)$ of $(1.2)$ on $\Sigma \times[0, \infty)$. Furthermore, there exists a subsequence of solutions which converges to a positive constant scalar curvature metric as $t_{j} \rightarrow \infty$.

In view of Proposition 2.2 below, we reduce the proof of our main Theorems to finding a uniformly lower bound of $\lambda(t)$ as in Section 2 .

Remark 1.2. Recently, we showed the global existence and convergence of solutions of the Calabi flow on Einstein 4-manifolds which is an extention of Proposition 1.1 to 4-dimensional manifolds ([Ch3]).

Acknowledgements. The author would like to express his thanks to Prof. S.-T. Yau for constant encouragement, Prof. B. Chow and the referee for valuable comments.

\section{§2. A uniformly lower bound}

For $g=e^{2 \lambda} g_{0}, R_{0}=R_{g_{0}}$, we have the following formulae for the quantities appearing in (1.2) and related ones:

$$
\begin{gathered}
R=R_{g}=e^{-2 \lambda}\left(R_{0}-2 \Delta_{0} \lambda\right) \\
\Delta R=e^{-2 \lambda} \Delta_{0} R, \quad \text { where } \Delta_{0}=\Delta_{g_{0}}, \Delta=\Delta_{g}, \\
d \mu=e^{2 \lambda} d \mu_{0}, \quad \text { where } d \mu_{0}=d \mu_{g_{0}}, d \mu=d \mu_{g} \\
\frac{\partial}{\partial t} d \mu=\triangle R d \mu \\
\int_{\Sigma} d \mu=\int_{\Sigma} e^{2 \lambda} d \mu_{0}=\int_{\Sigma} e^{2 \lambda_{0}} d \mu_{0}=\int_{\Sigma} d \mu_{0} .
\end{gathered}
$$

Remark 2.1. (2.5) implies the volume is fixed by the flow (1.2).

Then we have 
LEMMA 2.1. Under the flow (1.2), we have

$$
\int_{\Sigma} R^{2} d \mu \leq C\left(R_{0}, \lambda_{0}\right)
$$

for $0 \leq T \leq \infty$

In Chruściel's proof for Proposition 1.1, the crucial step is the so-called Bondi mass loss formula, i.e.

$$
\frac{d}{d t} \int_{\Sigma} e^{3 \lambda} d \mu_{0} \leq 0
$$

if the background metric $g_{0}$ has constant Gaussian curvature. Then, by using elliptic estimate and Moser inequality, he got a $C^{0}$-estimate. In general, this method does not work for any arbitrary background metric $g_{0}$.

Here we generalize Bondi mass loss formula to the case of surfaces $\left(\Sigma, g_{0}\right)$ with any arbitrary background metric $g_{0}$. In our situation, Chruściel's method can not be applied directly. The main difficulty for any arbitrary background metric $g_{0}$, the Bondi mass may not decay. Instead we follow the following alternative approach. First we get a kind of Harnack estimate $([\mathrm{Ch} 2])$ on the Bondi mass $\int_{\Sigma} e^{3 \lambda} d \mu_{0}$

$$
\begin{aligned}
\frac{d}{d t} \int_{\Sigma} e^{3 \lambda} d \mu_{0} \leq C_{1} & +C_{2} \int_{\Sigma} e^{-\lambda} d \mu_{0} \\
& -C_{3} \int e^{\lambda}\left[2\left|\nabla^{2} e^{-\lambda}\right|^{2}-\left(\Delta_{0} e^{-\lambda}\right)^{2}\right] d \mu_{0} .
\end{aligned}
$$

Then, from (2.6), one can show the next theorem.

Proposition 2.2. ([Ch2]) Let $\left(\Sigma, g_{0}\right)$ be a closed Riemann surface with any arbitrary background metric $g_{0}$. For any given smooth initial value $\lambda_{0}$, if

$$
\lambda(t) \geq-H
$$

for the positive constant $H$ which is independent of $t$, then there exists a smooth solution $\lambda(t)$ of $(1.2)$ on $\Sigma \times[0, \infty)$. Furthermore, there exists a subsequence of solution, say $\lambda\left(t_{j}\right)$, such that $g=e^{2 \lambda\left(t_{j}\right)} g_{0}$ converges to one of the constant curvature metric $g_{\infty}$ as $t_{j} \rightarrow \infty$. 
Remark 2.2. The similar results hold for the 3-dimensional Calabi flow. We refer to $[\mathrm{CW}]$ for details.

Hence, in view of Proposition 2.2, in order to show the main Theorems, all we need is to find a uniformly lower bound on $\lambda$. In the sequel, we will follow the notion as in [Ch2].

Definition 2.1. We say that $\lambda(t)$ satisfies the property $(*)$ if there is a point $x \in \Sigma$ and positive constants $\rho, \varepsilon, C$ such that, for $g=e^{2 \lambda} g_{0}$ the inequality

$$
\int_{B(x, \rho)} e^{-\varepsilon \lambda(t)} d \mu_{0} \leq C
$$

holds.

LEMMA 2.3. ([Ch2]) For a fixed conformal class $\left(\Sigma, e^{2 \lambda} g_{0}\right)$ where volume and $\int_{\Sigma} R^{2} d \mu$ are bounded and such that $\lambda$ satisfies the property $(*)$. Then there are positive constants $C_{0}$ and $\delta_{0}$ such that

$$
\int_{\Sigma} e^{-\delta_{0} \lambda} d \mu_{0} \leq C_{0}^{\prime} .
$$

As a consequence, there is a constant $C_{0}$ such that

$$
\lambda \geq-C_{0}
$$

In fact, for $h \geq 2$, one can show easily that

LEMMA 2.4. ([Ch2]) For a fixed conformal class $\left(\Sigma, e^{2 \lambda} g_{0}\right)$ where volume and $\int_{\Sigma} R^{2} d \mu$ are bounded and such that genus $h \geq 2$. Then $\lambda$ satisfies (2.8) and then has a uniformly lower bound.

Remark 2.3. In the case where $h=1$ and $h=0$, first we will show that $\lambda$ satisfies $(*)$ and then we can show (2.9) using the Lemma 2.3.

For $h=1$, we have the next lemma,

LEMMA 2.5. For a fixed conformal class $\left(\Sigma, e^{2 \lambda} g_{0}\right)$ where volume and $\int_{\Sigma} R^{2} d \mu$ are bounded and such that genus $h=1$. Then $\lambda$ satisfies $(*)$. 
Proof. Given $x \in \Sigma$, define the mass of $x$ by

$$
m(x)=\text { mass of } x=\lim _{\rho \rightarrow 0} \limsup _{t \rightarrow T} \int_{B(x, \rho)} e^{2 \lambda} d \mu_{0}
$$

and put

$$
E(x)=\lim _{\rho \rightarrow 0} \limsup _{t \rightarrow T} \int_{B(x, \rho)} R^{2} d \mu .
$$

Remark 2.4. A point $x \in \Sigma$ will have large mass $m(x)$ if $e^{\lambda}$ concentrates at $x$. On the other hand, if $m(x)$ is small enough, $e^{\lambda}$ will be bounded in a small neighborhood of $x$.

Following [G, Proposition 2.1] or [Chen], for $g \in\left[g_{0}\right]$ we put $g=e^{2 \lambda} g_{0}$. If $\int d \mu \leq V$ and $\int_{\Sigma} R^{2} d \mu \leq \beta^{2}$, for some positive constants $V, \beta$, then for a given $x \in \Sigma$, either one of the following holds:

$$
m(x)=0
$$

or

$$
m(x) \geq \frac{4 \pi}{E(x)} \geq \frac{4 \pi}{\beta^{2}} .
$$

Hence one has either $([\mathrm{CW}])$

(i)

$$
\max _{\Sigma} \lambda \leq C\left(\int d \mu, \int R^{2} d \mu\right)
$$

or that

(ii) there is a nonempty finite set $S=\left\{x_{1}, \ldots, x_{k}\right\}$ and a subsequence $\left\{t_{j}\right\}$ such that, given a compact set $K \subset \subset \widetilde{\Sigma}=\Sigma-S$,

$$
\max _{K} \lambda \leq C\left(K, \int d \mu, \int R^{2} d \mu\right) .
$$

Moreover, $w=\lim _{t_{j} \rightarrow T} \lambda$ is defined on $\widetilde{\Sigma}$ and the inequality

$$
w \leq C\left(\int d \mu, \int R^{2} d \mu\right)
$$

holds. 
Now with respect to $g_{0}$, from [C] and [Ch1, Lemma 3.2], we have the local Sobolev constant $A_{0}=A_{0}(n)$, i.e., for $\varphi=e^{\lambda / 2} f, \varphi \in C_{0}^{\infty}\left(B_{\rho}\right)$, $\rho<i_{0} / 2$,

$$
\left(\int_{B_{\rho}}|\varphi|^{2 l} d \mu_{0}\right)^{1 / l} \leq A_{0}\left[\left.\int_{B_{\rho}}||^{0} \varphi\right|^{2} d \mu_{0}\right],
$$

where $i_{0}$ is the injectivity radius with respect to $g_{0}$ and

$$
l= \begin{cases}n /(n-2), & n>2, \\ <\infty, & n=2 .\end{cases}
$$

Next we are able to estimate the local Sobolev constant with respect to $g$ in the fixed conformal class. For simplicity, we study only the case where $l=2$. Now for $B_{\rho} \subset K$ where $\max _{K} \lambda \leq C\left(K, \int d \mu, \int R^{2} d \mu\right)$ and $\varphi=e^{\lambda / 2} f, \varphi \in C_{0}^{\infty}\left(B_{\rho}\right), \rho<i_{0} / 2$, from (2.13) we have

$$
\begin{aligned}
\left(\int_{B_{\rho}}|f|^{4} d \mu\right)^{1 / 2} & =\left(\int_{B_{\rho}}|\varphi|^{4} e^{-2 \lambda} d \mu\right)^{1 / 2} \\
& =\left(\int_{B_{\rho}}|\varphi|^{4} d \mu_{0}\right)^{1 / 2} \\
& \leq A_{0}\left(\int_{B_{\rho}}|\nabla \varphi|^{2} d \mu_{0}\right) \\
& \leq\left.\left. C \int_{B_{\rho}} f^{2}\right|^{0} \lambda\right|^{2} e^{\lambda} d \mu_{0}+C \int_{B_{\rho}}\left|\nabla^{0} f\right|^{2} e^{\lambda} d \mu_{0} \\
& \leq C_{7} \int_{B_{\rho}} f^{2}\left|{ }^{0} \nabla\right|^{2} e^{\lambda} d \mu_{0}+C_{4} \int_{B_{\rho}}\left|{ }^{0} \nabla\right|^{2} d \mu_{0} .
\end{aligned}
$$

Now in order to have the local Sobolev constant bound as in (2.18) and (2.19), we need to get a suitable estimate as in (2.17) for $\int_{B_{\rho}} f^{2}\left|{ }^{0} \nabla\right|^{2} e^{\lambda} d \mu_{0}$ in (2.14).

In fact since $2 \Delta_{0} \lambda=R_{0}-e^{2 \lambda} R$ and $B_{\rho} \subset K$, we have

$$
\int_{B_{\rho}}\left(\Delta_{0} \lambda\right)^{2} d \mu_{0} \leq C+C \int_{B_{\rho}} R^{2} d \mu \leq C_{5} .
$$

Then

$$
\lambda-\bar{\lambda} \in W^{2,2}\left(B_{\rho / 2}\right) \subset W^{1, p}\left(B_{\rho / 2}\right),
$$


for any $p<\infty$ and $\bar{\lambda}=\int_{B_{\rho}} \lambda d \mu_{0} / \int_{B_{\rho}} d \mu_{0}$. Therefore we get

$$
\int_{B_{\rho / 2}}\left|\nabla^{0} \lambda\right|^{p} d \mu_{0} \leq C_{6}(p), \quad \text { for any } p<\infty .
$$

Let $E_{b}=\left\{x \in B_{\rho / 2}:||^{0} \lambda \mid(x) \geq b\right\}, b \gg 1$. The we get the estimate

$$
\begin{aligned}
\int_{B_{\rho / 2}} f^{2}|\stackrel{0}{\nabla} \lambda|^{2} e^{\lambda} d \mu_{0}= & \int_{E_{b}^{c}} f^{2}|\stackrel{0}{\nabla} \lambda|^{2} e^{-\lambda} d \mu+\int_{E_{b}} f^{2}\left|\nabla^{0} \lambda\right|^{2} e^{-\lambda} d \mu \\
\leq & b^{2}\left(\int_{B_{\rho / 2}} f^{4} d \mu\right)^{1 / 2}\left(\int_{B_{\rho / 2}} d \mu_{0}\right)^{1 / 2} \\
& +\left(\int_{B_{\rho / 2}} f^{4} d \mu\right)^{1 / 2}\left(\int_{B_{\rho / 2}}|\stackrel{0}{\nabla} \lambda|^{4} d \mu_{0}\right)^{1 / 2}
\end{aligned}
$$

From (2.15), we get for $p>4$, that

$$
C_{6} \geq \int_{E_{b}}\left|\nabla^{0} \lambda\right|^{p} d \mu_{0} \geq b^{p-4} \int_{E_{b}}\left|{ }^{0} \lambda\right|^{4} d \mu_{0}
$$

and then

$$
\int_{E_{b}}\left|\nabla^{0} \lambda\right|^{4} d \mu \leq C_{6} b^{4-p}
$$

From (2.16),

$$
\int_{B_{\rho / 2}} f^{2}\left|{ }^{0} \lambda\right|^{2} e^{2 \lambda} d \mu_{0} \leq\left[b^{2}\left(\int_{B_{\rho / 2}} d \mu_{0}\right)^{1 / 2}+\left(C_{6} b^{4-p}\right)^{1 / 2}\right]\left(\int_{E_{b}} f^{4} d \mu\right)^{1 / 2} .
$$

This and (2.14) imply

$$
\begin{aligned}
\left(\int_{B_{\rho / 2}}|f|^{4} d \mu\right)^{1 / 2} \leq C_{7} & {\left[b^{2}\left(\int_{B_{\rho / 2}} d \mu_{0}\right)^{1 / 2}+\left(C_{6} b^{4-p}\right)^{1 / 2}\right]\left(\int_{E_{b}} f^{4} d \mu\right)^{1 / 2} } \\
& +C_{4} \int_{B_{\rho / 2}}|\nabla f|^{2} d \mu_{0} .
\end{aligned}
$$

Choose $b$ large enough (depending on $p, A_{0}$ ) and $\int_{B_{\rho / 2}} d \mu_{0}$ small enough (depending on $b$ ). We can absorb the first term on the right-hand side of the above inequality into the left-hand side. On the other hand, for $n=2$ 
and $f \in C_{0}^{\infty}\left(B_{\rho / 2}\right)$, we have $\int_{B_{\rho / 2}}\left|\nabla^{0} f\right|^{2} d \mu_{0}=\int_{B_{\rho / 2}}|\nabla f|^{2} d \mu$, and hence we have

$$
\left(\int_{B_{\rho / 2}}|f|^{4} d \mu\right)^{1 / 2} \leq A_{0}^{\prime}\left(\int_{B_{\rho / 2}}|\nabla f|^{2} d \mu\right)
$$

for $B_{\rho / 2} \subset K$.

In general, for any $1<l<\infty, \varphi=e^{\lambda / l} f, \varphi \in C_{0}^{\infty}\left(B_{\rho}\right)$, doing the same trick as above, one can show that

$$
\left(\int_{B_{\rho / 2}}|f|^{2 l} d \mu\right)^{1 / l} \leq A_{0}^{\prime}\left(\int_{B_{\rho / 2}}|\nabla f|^{2} d \mu\right),
$$

for $B_{\rho / 2} \subset K$ with $\int_{B_{\rho / 2}} d \mu_{0}$ small enough.

Next we study the inequality

$$
-\Delta e^{-\lambda}=e^{-\lambda}\left(\Delta \lambda-|\nabla \lambda|^{2}\right) \leq \frac{1}{2} e^{-\lambda}\left(e^{-2 \lambda} R_{0}-R\right) .
$$

In the case where $R_{0}=0$ on $\Sigma$, we have

$$
-\Delta f \leq b f
$$

for $f=e^{-\lambda}$ and $b=\frac{1}{2}|R|$.

But

$$
\int f^{2} d \mu \leq C \quad \text { and } \quad \int b^{2} d \mu \leq C .
$$

From (2.20), (2.21) and (2.22), we can apply Moser iteration as in [CW, Section 3]) for $n=2$. It follows that, for $\int_{B_{\rho / 2}} d \mu_{0}$ small enough,

$$
\sup _{B_{\rho / 4}} e^{-\lambda} \leq C_{8}
$$

Hence $\lambda$ satisfies $(*)$.

In the case where $R_{0} \neq 0$, we may assume $R_{0}$ is negative on some $B_{\rho} \subset K$ with small enough $\int_{B_{\rho / 2}} d \mu_{0}$. Then the differential inequality (2.21) still holds. Again by the same method as above, we have

$$
\sup _{B_{\rho / 4}} e^{-\lambda} \leq C_{9}
$$

Now we have proved that $\lambda(t)$ satisfies $(*)$. 
For a fixed conformal class where volume and $\int_{\Sigma} R^{2} d \mu$ are bounded and such that $h=0$, one has

LEMma 2.6. For $\left(S^{2}, g_{0}\right)$ where volume and $\int_{\Sigma} R^{2} d \mu$ are bounded. If $R_{0} \geq 0, R_{0} \neq 0$ and $R_{0}=0$ in a ball $B\left(p_{0}, \rho_{0}\right)$, then $\lambda(t)$ does satisfy $(*)$.

Proof. As before we have

$$
-\Delta f \leq b f
$$

on $B\left(p_{0}, \rho_{0}\right)$ and $f=e^{-\lambda}, b=\frac{1}{2}|R|$. Then, based on the same arguments as in the previous lemma, we have

$$
\sup _{B_{\rho}} e^{-\lambda} \leq C_{10}
$$

for some ball $B_{\rho} \subset B\left(p_{0}, \rho_{0}\right)$.

It follows that $\lambda(t)$ satisfies $(*)$.

Then Theorem 1.3 and Theorem 1.4 follow easily from Lemma 2.3, Lemma 2.5, Lemma 2.6 and Proposition 2.2.

\section{REFERENCES}

[C] C. B. Croke, Some isoperimetric inequalities and eigenvalue estimates, Ann. Sci. Ec. Norm. Super., 13 (1980), 419-435.

[Ca1] E. Calabi, Extremal Kähler metrics, Seminars on Differential Geometry (S. T. Yau, ed.), Princeton Univ. Press and Univ. of Tokyo Press, Princeton, New York (1982), pp. 259-290.

[Ca2] E. Calabi, Extremal Kähler metrics II, Differential Geometry and Complex Analysis (I. Chavel and H. M. Farkas, eds.), Springer-Verlag, Berlin-Heidelberg-New York-Tokyo (1985), pp. 95-114.

[Ch1] S.-C. Chang, Critical Riemannian 4-manifolds, Math. Z., 214 (1993), 601-625.

[Ch2] S.-C. Chang, Global existence and convergence of solutions of Calabi flow on surfaces of genus $h \geq 2$, J. of Mathematics of Kyoto University, 40 (2000), no. 2, 363-377.

[Ch3] S.-C. Chang, Global existence and convergence of solutions of the Calabi flow on Einstein 4-manifolds, Nagoya Math. J., 163 (2001), 193-214.

[Ch4] S.-C. Chang, Recent developments on the Calabi flow, Contemporary Mathematics, 367 (2005), 17-42.

[Chen] X. X. Chen, Calabi flow in Riemann surfaces revisited: a new point of view, IMRN (2001), no. 6, 275-297. 
[Chru] P. T. Chruściel, Semi-global existence and convergence of solutions of the Robinson-Trautman (2-dimensional Calabi) equation, Commun. Math. Phys., 137 (1991), 289-313.

[CW] S.-C. Chang and J.-T. Wu, On the existence of extremal metrics for $L^{2}$-norm of scalar caurvature on closed 3-manifolds, J. of Mathematics of Kyoto University, 39 (1999), no. 3, 435-454.

[G] M. J. Gursky, Compactness of conformal metrics with integral bounds on curvature, Duke Math. J., 72 (1993), no. 2, 339-367.

[GT] D. Gilbarg and N. S. Trudinger, Elliptic partial differential equation of second order, Springer-Verlag, New York, 1983.

$[\mathrm{KW}]$ J. L. Kazdan and F. W. Warner, Existence and conformal deformation of metrics with prescribed Gaussian and scalar curvatures, Ann. of Math., 101 (2) (1975), $317-331$.

Department of Mathematics

National Tsing Hua University

Hsinchu

Taiwan 30043

R.O.C.

scchang@math.nthu.edu.tw 